\title{
How to Enhance the Employee Well-Being at Work?
}

\author{
Marko Divjak \\ DOBA Business School, Slovenia \\ marko.divjak@doba.si
}

\section{Živa Veingerl Čič}

DOBA Business School, Slovenia

ziva.veingerl-cic@doba.si

Purpose: This paper aims to evaluate the predictors of well-being in the workplace, psychological capital and mindfulness in particular.

\begin{abstract}
Study design/methodology/approach: The research was based on a convenient sample of 65 part-time online students at DOBA Business School. Two standardized questionnaires were administered online to gather the data: The Workplace PERMA Profiler and the Psychological Capital Questionnaire. Additionally, measures of stress perception and mindfulness practice were added to the survey instrument.
\end{abstract}

Findings: The results have shown that psychological capital, hope in particular, significantly predicts employee well-being, while the frequency of practising mindfulness does not improve the predictive power of the linear regression model.

Originality/value: The paper discusses the available measures and interventions and provides concrete guidelines for managers to enhance employee well-being in the workplace.

\section{Introduction}

According to Martin Seligman, the father of positive psychology, well-being is more than just feeling good and happy; it reflects the amount of flourishing and optimal psychosocial functioning. In his definition of well-being, Seligman combines the emotional (subjective) and psychological (eudemonic) paradigm of well-being. He proposed the PERMA model of wellbeing, which consists of five pillars: Positive emotion, Engagement, Relationships, Meaning and Accomplishment (Seligman, 2011). The element of Positive emotion is the most obvious connection to happiness. It is the ability to remain optimistic and view one's past, present, and future from a constructive perspective. When a person is engaged he is fully absorbed in a current momentum. This often happens while taking part in activities where one builds upon his strengths, which results in experiencing mastery, focus and enjoyment. Next, relationships and connections with significant others are crucial to meaningful lives. Having high-quality connections that are based on love, intimacy and emotional and physical bonds with other people is an important element of well-being. Meaning refers to a sense of purpose and direction in one's life, while Accomplishment refers to a sense of satisfaction and fulfilment as a consequence of achieving one's goals (Butler and Kern, 2016).

Each of these five pillars contributes to well-being. The amount of well-being is a combination of both subjective and objective measures. While Positive emotion is a purely subjective category, some other pillars have both subjective and objective components. For instance, a person may indeed believe (subjective perspective) he has meaning in his life, good relationships and high accomplishments, although his perceptions may not be fully consistent with reality (objective perspective) (Seligman, 2011).

Well-being seems to play a role in the prevention of and in the recovery of physical conditions and diseases and so possibly contributes to an increase in life expectancy (Vazquez, Hervas, Rahona and Gomez-Baya, 2009). Apart from its benefits for each individual, well-being also plays a central role in the workplace contributing to several positive organizational outcomes. The evidence shows that people who achieve good standards of well-being at work are likely 
to be more creative, more loyal, more productive, and provide better customer satisfaction than individuals with poor standards of well-being at work (New Economics Foundation, 2014). Also, employee well-being seems to increase the long term organizational success, productivity and employee engagement on the one side, while decreasing turnover, absenteeism and accident rates on the other side (Chou, Hecker and Martin 2012).

Several pieces of research have demonstrated a positive linkage of well-being with inner psychological resources, psychological capital in particular (Avey, Luthans, Smith, Palmer, 2015; Gautam, Ningthoujam, Singh, 2019; Kun and Gadanecz, 2019; Malekitabar, Riahi and Malekitabar, 2016; Rabenu, Yaniv and Elizur, 2017; Youssef Morgan and Luthans, 2015). Psychological capital is composed of four dimensions and has been defined as "an individual's positive psychological state of development and is characterized by: (1) having the confidence to take on and put in the necessary effort to succeed at challenging tasks (self-efficacy); (2) making a positive attribution about succeeding now and in the future (optimism); (3) persevering toward goals and, when necessary, redirecting paths to goals to succeed (hope); and (4) when beset by problems and adversity, sustaining and bouncing back and even beyond to attain success (resilience)" (Luthans, Youssef and Avolio, 2007, p. 3).

As employee well-being has been linked with organizational performance, the managers should attempt to redirect their endeavours towards improving well-being at work either directly or indirectly via its antecedents such as psychological capital. The managers should thus consider promoting one or more positive psychology interventions that are focused on helping employees to strengthen their personal resources, to flourish and take pride in their roles within the organisational system, to function to the best of their abilities and to develop a positive overall experience of work (New Economics Foundation, 2014). A positive psychology intervention is any intentional activity or method that is based on (a) the cultivation of positive subjective experiences, (b) the building of positive individual traits, or (c) the building of civic virtue and positive institutions (Meyers, van Woerkom, and Bakker, 2013). Research indicates that positive psychology interventions (e.g. interventions to develop/enhance psychological capital, gratitude exercises, cognitive-behavioural solution-based coaching, meditation exercises, resilience programs, cultivating realistic optimism, practising mindfulness) act as a promising tool for enhancing employee well-being and performance and for diminishing stress and burnout (Meyers et al., 2013; Salanova, Llorens and Martínez, 2016). The benefits of practising mindfulness, which could be considered as one of the efficient positive psychology interventions to enhance well-being in the workplace, have also been documented by Hülsheger, Alberts, and Lang (2013). They found out that practising mindfulness was negatively related to emotional exhaustion and positively related to job satisfaction.

This paper aimed to evaluate the predictors of well-being in the workplace, psychological capital and mindfulness in particular, among part-time (employed) online students and to discuss the measures and interventions to enhance employee well-being. In line with the theoretical background presented above, we have formulated the following research hypotheses:

H1: Psychological capital is significantly positively related to well-being.

$\mathrm{H} 2$ : The frequency of practising mindfulness is significantly positively related to well-being.

\section{Method}

\subsection{Participants}

The research was carried out among the part-time online students at DOBA Business School, who participated in the master course Positive Psychology for Modern Business Management 
during the academic year 2019/2020. Out of 108 course's participants, almost two-thirds decided to take part in the survey, which resulted in a convenient sample of $n=65$ respondents. The large majority of respondents is employed, 61 per cent of respondents are female and the mean age is $37,4(\mathrm{SD}=9,17)$. Approximately half of the respondents are Slovenes (52 per cent) and the other half is Croatians (48 per cent).

\subsection{Survey instruments}

A quantitative research strategy was adopted with a structured online questionnaire as a data collection technique. To measure subjective well-being, the Workplace PERMA Profiler was used. The original questionnaire designed by Kern (2014) was translated into Slovene and Croatian language. It consists of 15 items that converge into five PERMA pillars: Positive emotion, Engagement, Relationships, Meaning, Accomplishment. Each item is evaluated on a 10-point scale and the result for each pillar is calculated as an average score of the three corresponding items. A well-being total score is calculated as an average of the five PERMA pillars.

To measure psychological capital, the Psychological Capital Questionnaire, designed by Luthans, Avolio and Avey (2007), was used. The longer version of the questionnaire with 24 items was administered in the English language. The questionnaire measures four psychological capital dimensions: self-efficacy, hope, optimism and resilience. Each item is evaluated on a 6point scale and the result for each dimension is calculated as a sum of scores on all six items corresponding to a particular dimension.

In addition, participants assessed their stress perception in the professional and private domain and reported on the frequency of practising mindfulness. Two items were used to assess the perceived stressfulness of work and private life on a 5-point scale and only one item was used to measure practising mindfulness (answers ranging from "never" to "every day").

\subsection{Data collection}

The online survey questionnaire was made available to students in the virtual learning environment as part of the course materials. Students had to fill in the questionnaire as a starting point to prepare and finalize the assignments, however, not all students provided consent to use their data for research purposes, which resulted in a smaller sample. The complete anonymity of the respondents was assured throughout the research process. Data collection took place in May 2020.

\section{Results}

The average total score of well-being is quite high as it accounts for almost 80 per cent of the maximum value (table 1). Bivariate analysis showed there were no statistically significant differences in the total score of well-being across genders (Mann-Whitney $U=570,5 ; p=0,629$ ) and no statistically significant correlation of the total score of well-being with age (Spearman's rho $=0,128 ; \mathrm{p}=0,303)$. Comparison of the results across the five pillars of the PERMA model reveals only minor distinctions. The average scores range from approximately 75 to 85 per cent of the maximum value. Participants on average reported the highest score on Accomplishment and the lowest score on Positive emotion and Engagement. The scores on Accomplishment are far more homogeneous than the scores on all other pillars, which is evident from the lowest value of the standard deviation.

Table 1: Descriptive statistics for the five pillars and the total score of well-being

\begin{tabular}{|l|c|c|}
\hline Pillars & M & SD \\
\hline Positive emotion & 7,45 & 1,46 \\
\hline Engagement & 7,70 & 1,40 \\
\hline
\end{tabular}




\begin{tabular}{|l|l|l|}
\hline Relationships & 8,01 & 1,44 \\
\hline Meaning & 8,06 & 1,41 \\
\hline Accomplishment & 8,32 & 0,94 \\
\hline TOTAL SCORE & 7,91 & 1,08 \\
\hline
\end{tabular}

To evaluate the predictors of well-being, a hierarchical linear regression model was introduced with the total score of well-being as a dependant variable. Independent variables (predictors) were entered into the model in two steps. In step 1, four dimensions of psychological capital were defined as predictors and in step 2, measures of stress perception and the frequency of practising mindfulness were added into the model.

The multiple correlation coefficient of psychological capital with well-being accounts to $\mathrm{R}=$ $0,583(\mathrm{~F}(4,60)=7,704 ; \mathrm{p}=0,000)$, which means that the four dimensions of psychological capital explain around 29,5 percent of variability in well-being. The addition of measures of stress perception and mindfulness practice in step 2 does not significantly improve the predictive value of the linear regression model $(F$ change $(3,57)=0,146 ; p=0,932)$. It is evident from table 2 that Hope is the only statistically significant predictor of well-being. Hope is positively moderately related to well-being.

Table 2: Predictors of the total score of well-being

\begin{tabular}{|l|l|c|c|c|}
\hline \multirow{4}{*}{ Model 1} & Predictors & Beta & $\mathrm{t}$ & $\mathrm{p}$ \\
\cline { 2 - 5 } & Hope & 0,408 & 2,396 & 0,020 \\
\cline { 2 - 5 } & Optimism & $-0,099$ & $-0,764$ & 0,448 \\
\cline { 2 - 5 } & Resilience & 0,225 & 1,575 & 0,120 \\
\cline { 2 - 5 } & Self-efficacy & 0,078 & 0,492 & 0,625 \\
\hline Model 2 & Hope & 0,384 & 2,078 & 0,042 \\
\cline { 2 - 5 } & Optimism & $-0,098$ & $-0,713$ & 0,479 \\
\cline { 2 - 5 } & Resilience & 0,233 & 1,569 & 0,122 \\
\cline { 2 - 5 } & Self-efficacy & 0,064 & 0,386 & 0,701 \\
\cline { 2 - 5 } & How would you rate the nature of work in your workplace?* & 0,088 & 0,618 & 0,539 \\
\cline { 2 - 5 } & How would you rate your life in the last 30 days? & $-0,029$ & $-0,204$ & 0,839 \\
\cline { 2 - 5 } & How often have you been practising mindfulness in the last 12 & 0,016 & 0,144 & 0,886 \\
& months? & & \\
\hline
\end{tabular}

*Note: answers were given on a 5-point scale (1 - not stressful at all; 5 - very stressful).

\section{Discussion}

The paper aimed to investigate the predictive power of psychological capital and mindfulness in explaining employee well-being at work. The results have shown that psychological capital explains around 30 per cent of the variance in well-being. Among the four dimensions of psychological capital, only Hope seems to significantly predict employee well-being. In other words, respondents with stronger perseverance and motivation for goal attainment and with more flexibility in redirecting their paths and means towards goals when necessary, are more likely to report higher levels of employee well-being. This finding is partly consistent with the results of Kun and Gadanecz (2019), who reported workplace well-being to be significantly correlated with all dimensions of psychological capital, among which the correlations with hope and optimism were the strongest. All in all, we can conclude that psychological capital predicts employee well-being at work, which supports the hypothesis $\mathrm{H} 1$ and is in line with the findings of previous research (Avey et al., 2015; Gautam et al., 2019; Kun and Gadanecz, 2019; Malekitabar et al., 2016; Rabenu et al., 2017; Youssef Morgan and Luthans, 2015).

When additional variables (measures of stress perception and mindfulness practice) were added to the model, the predictive power of the linear regression model did not significantly improve. It seems that the frequency of practising mindfulness is not related to employee well-being, which contradicts the findings of previous research (Hülsheger et al., 2013; Meyers et al., 2013; Salanova et al., 2016). The insignificant correlation of mindfulness practice with well-being 
could be at least to a certain extent explained by the methodological shortcomings of this research. Apart from the small-size sample, the variability in the frequency of practising mindfulness seems low as more than half of the respondents never practice mindfulness and another 15 per cent of respondents practice it only a few times a year. For the mindfulness practice to exert positive outcomes, one needs to practice it regularly, at least weekly. Also, for those respondents who reported practising mindfulness, details about the type and quality of adopted mindfulness exercises remain unknown. To learn more about the benefits of mindfulness for employee well-being, either an experimental or quasi-experimental research design would be required. Such a design would enable a more systematic investigation of the impacts of a structured mindfulness intervention, possibly implemented and carried out at work during working hours. To conclude, the results of this paper do not support hypothesis $\mathrm{H} 2$ about the frequency of practising mindfulness being significantly positively related to employee wellbeing at work.

To enhance employee well-being at work and consequently contribute to positive organizational performance, both employees and managers should focus on strengthening the components of well-being directly, e.g. by experiencing more positive emotions, using signature character strengths to boost engagement, stimulating high-quality relationships at work, helping employees to understand the meaning and purpose of their work and enabling them to experience success, and indirectly via its antecedents, such as hope. To strengthen hope, one should attempt to set up challenging but achievable goals, break down complex goals into several simpler concrete tasks and think about alternative paths for goal achievement in advance. A key takeaway message for the managers is to be careful with the amount of workload (an employee needs to believe that the workload is manageable and the goals achievable) and to discuss the means and paths for goal attainment together with employees, especially for the more difficult and complex goals (an employee needs to have an idea and direction of how to accomplish the task).

This research is based on a small non-random sample, which is one of its major shortcomings and limits the possibility of generalizing the findings beyond the research context. Future research should attempt to address a more representative sample of employees to be able to draw more solid conclusions about the predictors and antecedents of well-being at work. One suggestion for future research is to investigate the predictive value of additional antecedents of employee well-being, apart from psychological capital and mindfulness practice, for instance, elaborated measures of stress exposure perception or the strategies for coping with stress at work.

\section{References}

Avey, J. B., Luthans, F., Smith, R. M., Palmer, N. F. (2010). Impact of Positive Psychological Capital on Employee Well-Being Over Time. Journal of Occupational Health Psychology 15(1), 17-28.

Butler, J., Kern, M. L. (2016). The PERMA-Profiler: A brief multidimensional measure of flourishing. International Journal of Wellbeing, 6(3), 1-48.

Chou, H. Y., Hecker, R., Martin, A. (2012). Predicting nurses' well being from job demands and resources: A cross sectional study of emotional labour. Journal of Nursing Management, 20, 502-511.

Gautam, V., Ningthoujam, S., Singh, T. (2019). Impact of Psychological Capital on Well-Being of Management Students. Theoretical Economics Letters, 9, 1246-1258.

Hülsheger, U. R., Alberts, H. J. E. M., Feinholdt, A., Lang, J. W. B. (2013). Benefits of mindfulness at work: The role of mindfulness in emotion regulation, emotional exhaustion, and job satisfaction. Journal of Applied Psychology, 98, 310-325

Kern, M. L. (2014). The Workplace PERMA Profiler. http://www.peggykern.org/uploads/5/6/6/7/56678211/workplace_perma_profiler_102014.pdf 
Kun, A., Gadanecz, P. (2019). Workplace happiness, well-being and their relationship with psychological capital: A study of Hungarian teachers. Current Psychology. https://doi.org/10.1007/s12144-019-00550$\underline{0}$.

Luthans, F., Avolio, B. J., Avey, J. B. (2007). Psychological Capital (PsyCap) Questionnaire. Menlo Park, USA: Mind Garden, Inc.

Luthans, F., Youssef, C. M., Avolio, B. J. (2007). Psychological capital. New York: Oxford University Press.

Malekitabar, M., Riahi, M., Malekitabar, A. R. (2016). The Role of Psychological Capital in Psychological Well-Being and Job Burnout of High Schools Principals in Saveh, Iran. Iranian Journal of Psychiatry and Behavioral Sciences, 11(1), e4507. https://dx.doi.org/10.5812/ijpbs.4507.

Meyers, M. C., van Woerkom, M., Bakker, A. B. (2013). The added value of the positive: A literature review of positive psychology interventions in organizations. European Journal of Work and Organizational Psychology, 22(5), 618-632.

New Economics Foundation. (2014). Well-being at work. A review of the literature. https://b.3cdn.net/nefoundation/71c1bb59a2ce151df7 8am6bqr2q.pdf

Rabenu, E., Yaniv, E., Elizur, D. (2017). The Relationship between Psychological Capital, Coping with Stress, Well-Being, and Performance. Current Psychology, 36, 875-887. https://doi.org/10.1007/s12144-0169477-4.

Salanova, M., Llorens, S., Martínez, I. M. (2016). Contributions from positive organizational psychology to develop healthy and resilient organizations. Psychologist Papers, 37(3), 177-184.

Seligman, M. (2011). Flourish: A Visionary New Understanding of Happiness and Well-Being. Free Press.

Vazquez, C., Hervas, G., Rahona J. J., Gomez-Baya, D. (2009). Psychological well-being and health. Contributions of positive psychology. Annuary of Clinical and Health Psychology, 5, 15-27.

Youssef Morgan, C. M., Luthans, F. (2015). Psychological capital and well being. Stress and Health: Journal of the International Society for the Investigation of Stress, 31(3), 180-188. https://doi.org/10.1002/smi.2623. 